\title{
A fluorescence correlation spectroscopy study of macromolecular tracer diffusion in polymer solutions
}

\author{
Ute Zettl $^{1}$, Matthias Ballauff ${ }^{2}$, Ludger Harnau ${ }^{3}$ \\ ${ }^{1}$ Physikalische Chemie I, University of Bayreuth, 95440 Bayreuth, Germany \\ ${ }^{2}$ Soft Matter and Functional Materials, Helmholtz-Zentrum Berlin, 14109 \\ Berlin, Germany \\ ${ }^{3}$ Max-Planck-Institut für Metallforschung, Heisenbergstr. 3, 70569 Stuttgart, \\ Germany, and Institut für Theoretische und Angewandte Physik, Universität \\ Stuttgart, Pfaffenwaldring 57, 70569 Stuttgart, Germany \\ E-mail: harnau@fluids.mpi-stuttgart.mpg.de
}

\begin{abstract}
We discuss the manner in which the dynamics of tracer polystyrene chains varies with the concentration of matrix polystyrene chains dissolved in toluene. Using fluorescence correlation spectroscopy and theory, it is shown that the cooperative diffusion coefficient of the matrix polystyrene chains can be measured by fluorescence correlation spectroscopy in the semidilute entangled concentration regime. In addition the self-diffusion coefficient of the tracer polystyrene chains can be detected for arbitrary concentrations. The measured cooperative diffusion coefficient is independent of the molecular weight of the tracer polystyrene chains because it is a characteristic feature of the transient entanglement network.
\end{abstract}




\section{Introduction}

The dynamics of solutions composed of high molecular weight polymer chains differs qualitatively from the dynamics of simple fluids due to entanglements. At a microscopic scale, entanglements arise from the fact that linear polymer chains are one-dimensionally connected objects which cannot cross each other. The resulting topological interaction strongly affects dynamical properties since it imposes constraints on the motion of the polymer chains. The common interpretation of the physical origin of entanglement phenomena is that a transient network emerges due to the interactions between the polymer chains. The so-called reptation model is the most developed and widely applied phenomenological theory for the motion of polymer chains (see e.g., ref [1] and references therein). It focusses on the motion of a single polymer chain in an static field due to the surrounding polymer chains. Although widely accepted, there are significant discrepancies between predictions of the reptation model and experiments on polymer solutions because the surrounding polymer chains due not act as a static field but exhibit themselves as cooperative fluctuations. Hence there is an important coupling between the single polymer chain motion and the cooperative network fluctuations. As a result of this coupling it should be possible to detect both the single polymer and the cooperative dynamics using the same experimental technique.

Recently it has been demonstrated that two different diffusion coefficients can be obtained with fluorescence correlation spectroscopy (FCS) using single-labeled polystyrene (PS) solutions 2]. The self-diffusion coefficient $D_{s}(c)$ results from FCS in the limit of small concentrations of labeled PS chains and for arbitrary concentrations $c$ of unlabeled PS chains. Moreover, the cooperative diffusion coefficient $D_{c o}(c)$ becomes accessible in the semidilute entangled regime due to an effective long-range interaction in the transient entanglement network. The self-diffusion coefficient describes the motion of one molecule relative to the surrounding molecules due to thermal motions while the cooperative diffusion coefficient describes the dynamics of a number of molecules in a concentration gradient. It has been pointed out that measurements of both $D_{s}(c)$ and $D_{c o}(c)$ are very interesting since a central problem in the dynamics of semidilute entangled polymer solutions is the quantitative understanding of the interplay of self-diffusion and cooperative diffusion. Motivated by this prospect, we therefore extend our previous study [2] to the case of macromolecular tracer diffusion as is illustrated in figure 1. Hence we study the dynamics of long end-labeled tracer PS chains (black wriggled lines in figure 1) immersed in a polymer solution consisting of shorter matrix PS chains (gray wriggled lines in figure 1). The study is devoted to an understanding of the coupling of self- and cooperative motion due to topological constraints. Varying the concentration and the molecular weight of the matrix PS chains allows us to modify these topological constraints.

\section{Dye labeled tracer polystyrene chains}

Linear PS chains with different molecular weights and low polydispersity were prepared as discussed in ref. 3]. Our samples were composed of a small concentration (around $10^{-8} \mathrm{M}$ ) of Rhodamine B labeled tracer PS chains of molecular weight $M_{w}^{(t r)}$ in toluene solutions in which matrix PS chains of molecular weight $M_{w}^{(m a)}$ were dissolved at various concentrations. The resulting systems are denoted as PS- $M_{w}^{(t r)} / M_{w}^{(m a)}$ as is 


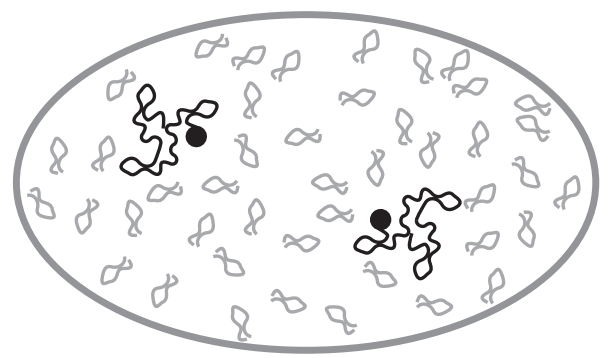

Figure 1. Schematic illustration of a polymer solution composed of a small concentration of labeled tracer polymer chains (black wriggled lines) and matrix polymer chains of different molecular weight (gray wriggled lines). Each labeled polymer chain carries only one dye molecule at one of its ends which is marked by a black dot. The FCS observation volume is enclosed by the thick gray ellipsoidal line. The size of the polymer chains, the size observation volume, and the number of polymer chains are not drawn to absolute scale. Only the fact that the molecular weight of the matrix polymer chains can be different from that of the tracer polymer chains is relevant.

indicated in table 1 Each labeled PS chain carries only one dye molecule at one of its ends. We have used preparative gel permeation chromatography to separate labeled polymer chains and free dye molecules [3, 4. Therefore, the resulting PS solutions do not contain any measurable amount of free dye molecules.

Table 1. Molecular weights $M_{w}^{(t r)}$ and $M_{w}^{(m a)}$ of the tracer and matrix PS chains, respectively. The concentration at which the fast diffusion process appears in the FCS measurements is denoted as $c^{+}$. In the main text and the figures the various PS solutions are denoted by the names given in the first column of the table. For comparison the overlap concentration $c^{\star}$ of the matrix PS chains is shown in the last column. [5

\begin{tabular}{lllll}
\hline Name & $M_{w}^{(t r)}[\mathrm{kg} / \mathrm{mol}]$ & $M_{w}^{(m a)}[\mathrm{kg} / \mathrm{mol}]$ & $c^{+}[\mathrm{wt} \%]$ & $c^{\star}[\mathrm{wt} \%]$ \\
\hline PS-17/17 & 17 & 17 & & $3.5 \pm 0.3$ \\
PS-264/17 & 264 & 17 & & $3.5 \pm 0.3$ \\
\hline PS-67/67 & 67 & 67 & 20.0 & $1.4 \pm 0.2$ \\
PS-264/67 & 264 & 67 & 20.0 & $1.4 \pm 0.2$ \\
\hline PS-264/264 & 264 & 264 & 6.5 & $0.54 \pm 0.12$ \\
\hline
\end{tabular}

\section{Fluorescence correlation spectroscopy}

FCS is a method relying on the detection and temporal analysis of the fluorescence signal emitted from a small confocal detection volume (see e.g., refs. 6, 7]). A laser beam is focused by an objective with high numerical aperture and excites fluorescent molecules entering the illuminated observation volume. Our FCS setup is based on the commercial ConfoCor2 setup (Carl Zeiss, Jena, Germany) [7] with a 40× Plan Neofluar objective characterized by the numerical aperture $\mathrm{NA}=0.9$. Fluorescence is excited by a He Ne-Ion laser at a wavelength of $543 \mathrm{~nm}$. For details of the FCS-measurements see refs. $[3,5,7,8]$. 
The emitted fluorescent light is detected by an avalanche photo diode. The time dependent intensity fluctuations are analyzed by an autocorrelation function $G(\tau, c)$, where $\tau$ denotes the time. In order to account for the possibility of the contribution of both self-diffusion and cooperative diffusion, the function

$$
G(\tau, c)=\sum_{i \in\{s, c o\}} G_{i}(0, c)\left(1+\frac{4 D_{i}(c) \tau}{w_{x, y}^{2}}\right)^{-1}\left(1+\frac{4 D_{i}(c) \tau}{w_{z}^{2}}\right)^{-1 / 2}
$$

is used to describe the experimental data within a coupled-mode model (see ref. 2 and references therein). Here $w_{x, y}=296 \mathrm{~nm}$ is the dimension of the observation volume perpendicular to the optical axis and $w_{z}=8 w_{x, y}$ is the dimension along the optical axis. $G_{i}(0, c)$ characterizes the contribution of the $i$-th component to the total amplitude $G(0, c)$ of the autocorrelation function. In equation (11) normal diffusion with mean square displacements $\phi_{i}(\tau, c)=6 D_{i}(c) \tau$ has been assumed. Deviations from normal diffusion are due to internal chain motions (see e.g., refs. [2, 9, 10, 11, 12, 13] and references therein) or due to molecular crowding (see e.g., refs. 2, 14, 15, 16, 17, 18, 19] and references therein).

FCS is not only sensitive to intensity fluctuations due to the motion of labeled molecules but also due to photokinetic processes of the fluorescent dyes which occur for short times $\tau<5 \times 10^{-3} \mathrm{~ms}$. This additional relaxation has been taken into account as discussed in refs. [3, 4, 5].

\section{Autocorrelation functions measured by FCS}

Two different types of macromolecular tracer diffusion behavior were obtained depending on the concentration and the molecular weight of the matrix polymer chains, as illustrated in figure 2, For the PS-264/17 sample containing short matrix PS chains $\left(M_{w}^{(m a)}=17 \mathrm{~kg} / \mathrm{mol}\right.$, see table 1), the measured autocorrelation function is characterized by a single self-diffusion process for arbitrary concentrations (open squares in figures 2 (a) - (d)). With increasing concentration the decay of the autocorrelation function shifts to longer times. The simple self-diffusion model given by equation (11) with $G_{s}(0, c)=1$ and $G_{c o}(0, c)=0$ is successful in describing the autocorrelation data for such systems. This holds also for solutions containing longer matrix PS chains for low concentrations (PS-264/67 in figures 2 (a), (b) and PS264/264 in figure 2 (a)). However, for higher concentrations, the simple self-diffusion model no longer describes the data in the case of the longer matrix PS chains. The autocorrelation data exhibit a second decay time on a shorter time scale (solid circles in figures 2 (c), (d) and crosses in figures 2 (b) - (d)). The experimental data can be described taking into account both self-diffusion and cooperative diffusion in equation (11), i.e., $G_{c o}(0, c) \neq 0$. The concentration $c^{+}$at which the second fast diffusion process is detected depends on the molecular weight of the matrix PS chains. Upon decreasing the molecular weight of the matrix PS chains the concentration $c^{+}$decreases (see table 1). In the case of the short matrix PS chains of molecular weight $M_{w}^{(m a)}=17 \mathrm{~kg} / \mathrm{mol}$ no second diffusion process has been observed as already mentioned above. As the entanglement molecular weight of PS is $18 \mathrm{~kg} / \mathrm{mol}$, entanglements of matrix polymer chains are not possible at any concentration of the short matrix PS chains of molecular weight $17 \mathrm{~kg} / \mathrm{mol}[20$.

An important result of the analysis of the autocorrelation functions for various concentrations is that the concentration $c^{+}$is independent of the molecular weight 
of the tracer PS chains. For example, $c^{+}=20.0 \mathrm{wt} \%$ for both the PS- $67 / 67$ and the PS-264/67 sample (see table 1). Moreover, only self-diffusion can be observed for both the PS-17/17 and the PS-264/17 sample, i.e., no concentration $c^{+}$can be defined for these samples (see table 11). Hence the fast diffusion process is not a characteristic property of the tracer PS chains but is related to the dynamics of the surrounding matrix PS chains as will be discussed in more detail in the next section.
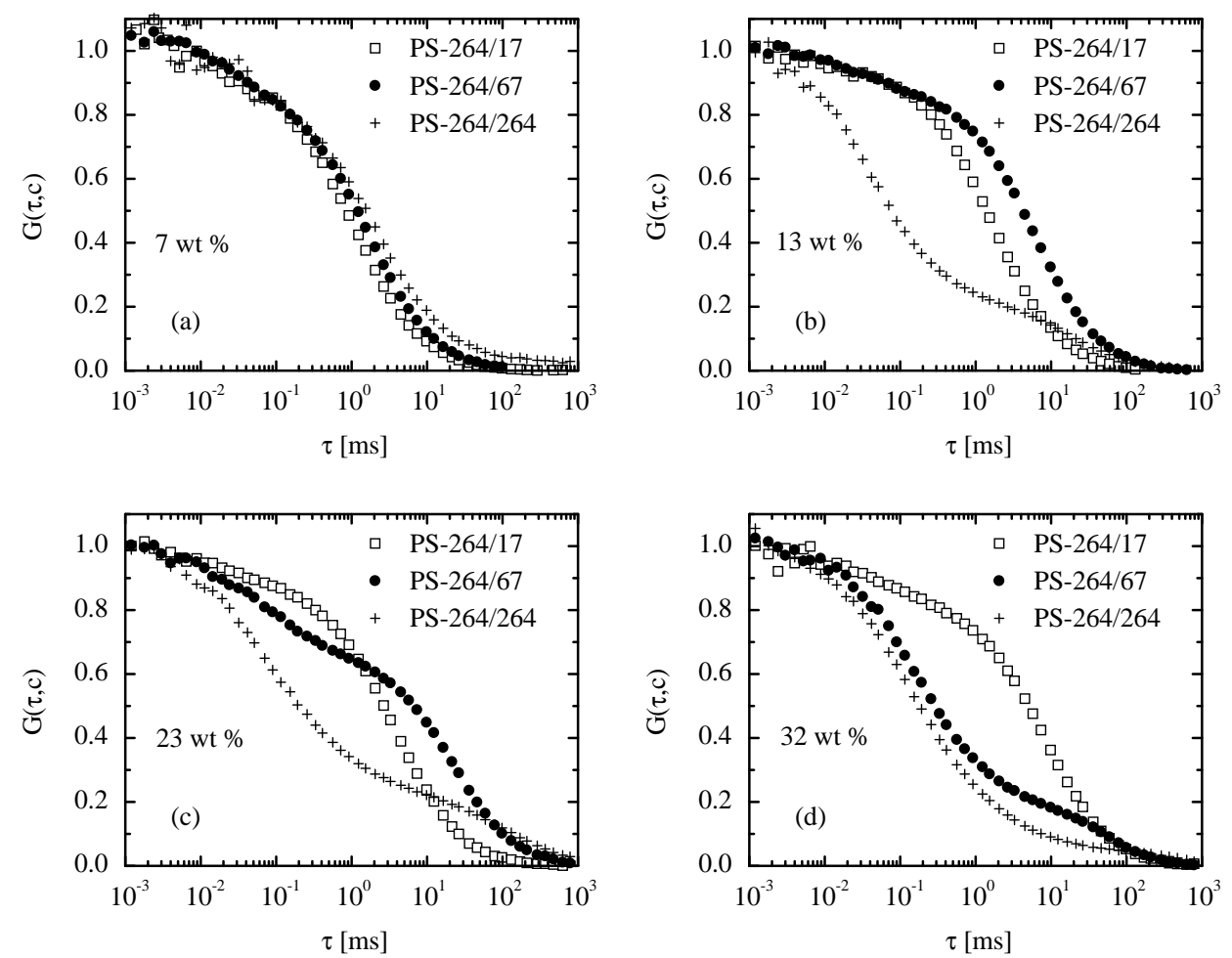

Figure 2. Normalized autocorrelation functions obtained from FCS for the three samples PS-264/17, PS-264/67, and PS-264/264 (see table11). The concentration of the matrix PS chains increases from (a) to (d) according to 7, 13, 23, $32 \mathrm{wt} \%$.

\section{Langevin and integral equation theory}

In this section we outline some points on the basic description of the cooperative dynamics of polymer solutions in terms of a Langevin and integral equation theory. The application of this approach to PS chains in solution has been discussed earlier [2, 21, 22]. Therefore, we only present the equations necessary for this study. The central assumption is that the total dynamic scattering function $S_{t o t}(q, c, \tau)$ is a good variable for characterizing the cooperative dynamics of the system, where $q$ is the absolute value of the scattering vector. The time evolution of $S_{t o t}(q, c, \tau)$ is assumed to be governed by a Langevin equation [1]. The total dynamic scattering function can be measured by dynamic light scattering (DLS). The cooperative diffusion coefficient $D_{c o}(c)$ determines the decay rate of the total dynamic scattering function and is given 
by

$$
D_{c o}(c) \stackrel{q \rightarrow 0}{=} \frac{k_{B} T}{4 \pi^{2} \eta} \int_{0}^{\infty} d q_{1} \frac{S_{t o t}\left(q_{1}, c, 0\right) q_{1}^{2}}{S_{t o t}(q, c, 0) q^{2}}\left(\frac{q_{1}^{2}+q^{2}}{2 q_{1} q} \log \left|\frac{q_{1}+q}{q_{1}-q}\right|-1\right)
$$

where the temperature $T$ and the viscosity $\eta$ characterize the solvent. Furthermore, the total static scattering function reads

$$
S_{t o t}(q, c, 0)=1+\bar{v} \operatorname{ch}(q, c) /\left(V_{p} P(q, c)\right),
$$

where $V_{p}$ is the volume of a dissolved polymer chain, $h(q, c)$ is a particle-averaged total correlation function, and $\bar{v}=0.916 \mathrm{~cm}^{3} / \mathrm{g}$ is the specific weight of PS [23]. The particle-averaged intramolecular correlation function $P(q, c)$ characterizes the geometric shape of the polymer chains at a given concentration c. The overall size of the polymer chains is reduced considerably upon increasing the concentration implying a concentration dependence of the particle-averaged intramolecular correlation function. Therefore, we consider the following particle-averaged intramolecular correlation function [24]

$$
P(q, c)=\left(1+0.549 q^{2} r_{g}^{2}(c)\right)^{-5 / 6}
$$

with the concentration dependent radius of gyration

$$
r_{g}^{2}(c)=r_{g}^{2}(0)\left(\Theta\left(c^{\star}-c\right)+\left(\frac{c}{c^{*}}\right)^{-1 / 8} \Theta\left(c-c^{\star}\right)\right) .
$$

Here $\Theta(x)$ is the Heaviside step function which is 1 for $x>0$ and zero elsewhere. Moreover, the overlap concentration $c^{\star}$ is the boundary concentration between the dilute and semidilute regimes (see table 1). This overlap concentration depends on the molecular weight according to $c^{\star} \sim\left(M_{w}^{(m a)}\right)^{-4 / 5}$, and has been determined for the PS solutions under considerations using FCS [5]. In addition the scaling law given $c^{-1 / 8}$ in equation (5) has been confirmed experimentally for PS in a good solvent using small angle neutron scattering [25]. The particle-averaged total correlation function is related to a particle-averaged direct correlation function $C(q, c)$ by the generalized Ornstein-Zernike equation of the Polymer Reference Interaction Site Model (PRISM), which reads (see e.g., refs [21, 26] and references therein)

$$
h(q, c)=P^{2}(q, c) C(q, c) /\left(1-\bar{v} c C(q, c) P(q, c) / V_{p}\right) .
$$

This generalized Ornstein-Zernike equation is supplemented by the Percus-Yevick approximation to account for steric effects [21. The osmotic pressure $p(c)$ is evaluated from equations (3) - (6) as

$$
p(c)=k_{B} T \bar{v} \int_{0}^{c} d c^{\prime} S_{t o t}\left(q, c^{\prime}, 0\right) / V_{p} .
$$

The PRISM integral equation theory has been successfully applied to various polymer solutions (see e.g., refs [21, 22, 27, 28]).

Figures $3(\mathrm{a}),(\mathrm{b})$, and (c) display the calculated cooperative diffusion coefficients of the 17, 67, and $264 \mathrm{~kg} / \mathrm{mol}$ matrix PS chains (solid lines) together with the experimental data measured with DLS (open squares) [2] and FCS (solid squares). The figures demonstrate that the measured cooperative diffusion coefficients agree with the calculated results as obtained from equations (2) - (6). In particular, the crossover region between the dilute and the semidilute regimes is captured correctly by 
the Langevin and integral equation theory. The maximum of the cooperativ diffusion coefficient in the semidilute entangled regime marks the onset of glassy dynamics. This friction controlled dynamics is not captured by equation (2). Therefore, deviations between the solid lines and the symbols are found for high concentrations in figure 3 Nevertheless, one can conclude from figures 3 (b) and (c) that the diffusion coefficient $D_{c o}(c)$ as obtained by FCS (solid squares) is indeed the cooperative diffusion coefficient of the matrix PS chains. The topological interactions in the semidilute solutions lead to coherent movements of matrix and tracer PS chains characterized by the cooperative diffusion coefficient $D_{c o}(c)$. The resulting temporal fluctuations of the detected fluorescence intensity can be measured by FCS even in the case that the number of labeled tracer PS chains $N_{l}$ is considerably smaller than the number of matrix PS chains $N_{m a}$, i.e., $N_{l} \approx N_{m a} \times 10^{-6}$ for the systems under consideration.
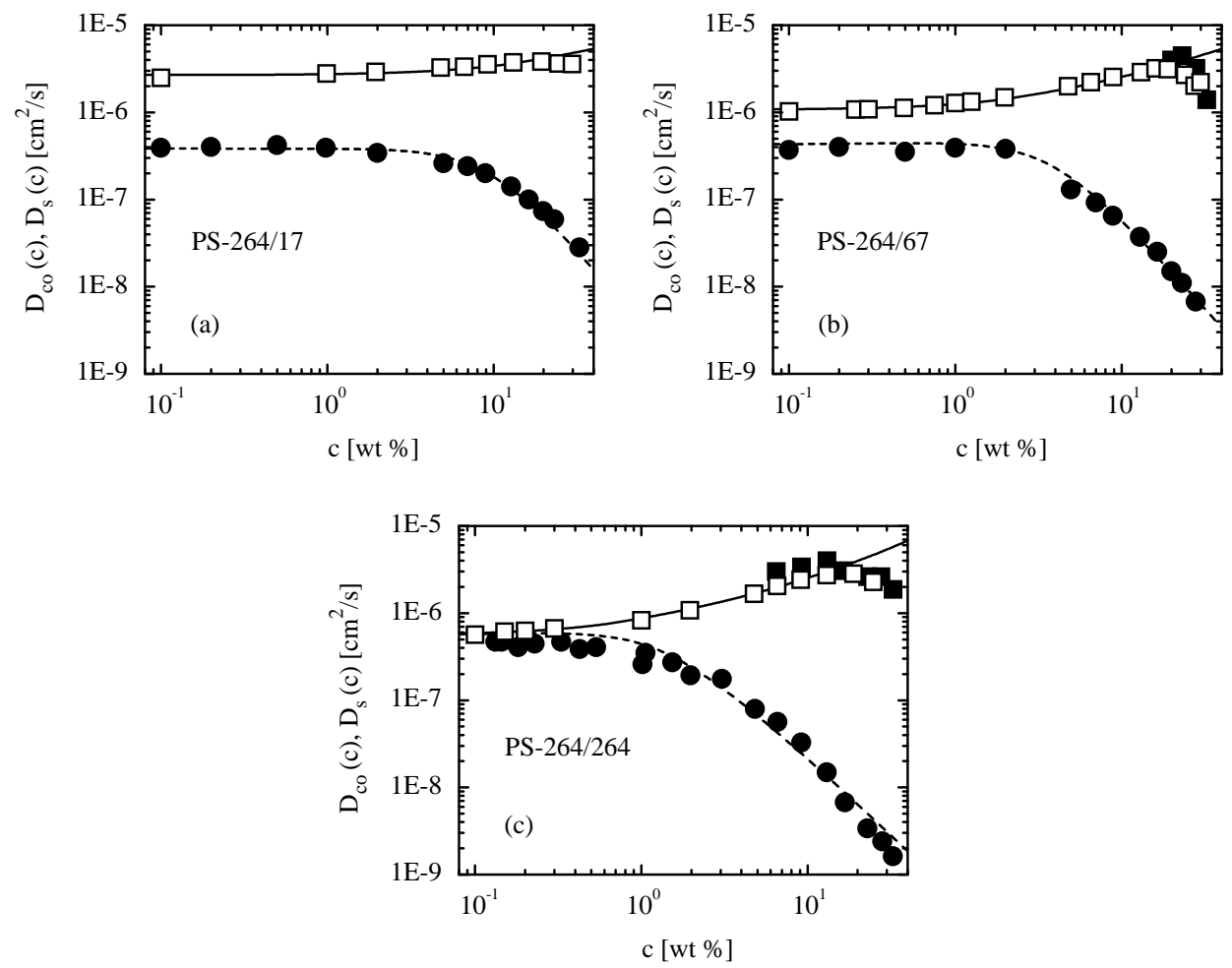

Figure 3. Cooperative diffusion coefficients $\left(D_{c o}(c)\right.$, squares $)$ and self-diffusion coefficients $\left(D_{s}(c)\right.$, circles) for the three samples PS-264/17, PS-264/67, and PS264/264 (see table 1) in panel (a), (b), and (c), respectively. Solid symbols refer to FCS measurements (see figure 21). The open squares denote DLS data obtained from the samples PS-17/17, PS-67/67, and PS-264/264 2]. The solid lines display the collective diffusion coefficients as obtained from the Langevin and integral equation theory according to equations (2) - (6). Dashed lines represent the calculated results as obtained from equation (8) with equations (2) and (7) as input and $\alpha=7.2,2.5,1$ in panel (a), (b), and (c), respectively.

The self-diffusion coefficient $D_{s}(c)$ as obtained using FCS measurements (see figure 2) and equation (1) are also shown in figure 3 (solid circles). $D_{s}(c)$ is found to decrease with increasing concentration of the matrix PS chains due to the friction 
between the polymer chains. The dashed lines in figure 3 are theoretical values calculated according to 29 ]

$$
\frac{D_{c o}(c)}{D_{s}(c)}=\alpha(1-\bar{v} c) \frac{d p(c)}{d c}
$$

with both $D_{c o}(c)$ and $p(c)$ obtained from the Langevin and integral equation theory given by equations (2) - (6). As a new feature of the present evaluation, we have introduced the parameter $\alpha$ in equation (8). This parameter describes the difference of the system under consideration from a solution consisting of matrix polymer chains and tracer chains of the same molecular weight, that is, $\alpha=1$. The dashed line in figure 3 (c) demonstrates that the measured self-diffusion coefficient of the solution PS-264/264 can be described by equation (8) with $\alpha=1$ and the Langevin and integral equation theory as input. Similarly, $D_{s}(c)$ can be calculated in agreement with experimental data for the samples PS-17/17 and PS-67/67 using $\alpha=1$ (data not shown). In order to describe the self-diffusion coefficients of the PS-264/67 and PS-264/17 samples, values of $\alpha=2.5$ and $\alpha=7.2$ above unity had to be chosen (dashed lines in figures 3 (b) and (a). The values of $\alpha$ different from unity reflect the fact that the molecular weights of the matrix and tracer polymer chains are different in the case of the samples PS-264/67 and PS-264/17.

Scaling arguments for self-avoiding random coils lead to the prediction $\alpha=$ $\left(M_{w}^{(m a)} / M_{w}^{(t r)}\right)^{-3 / 5}$, where the Flory exponent $\nu=3 / 5$ for a good solvent has been used. Hence one obtains $\alpha=2.3$ and $\alpha=5.2$ for the samples PS-264/67 and PS$264 / 17$, respectively. The predicted value $\alpha=2.3$ is close to the value $\alpha=2.5$ used in our analysis in the case of the PS-264/67 sample. This agreement confirms our earlier finding that the self-diffusion coefficients of both $264 \mathrm{~kg} / \mathrm{mol}$ PS chains and 67 $\mathrm{kg} / \mathrm{mol}$ PS chains fulfil scaling relations 2. However, the $17 \mathrm{~kg} / \mathrm{mol}$ PS chains are too short to be considered as self-avoiding random coils. Molecular stiffness leads to a more pronounced dependence of dynamical properties on the molecular weight than in the case of self-avoiding random coils 9 , 10, 11. Therefore, the value $\alpha=7.2$ used in our analysis is larger than $\alpha=5.2$.

Figure 4 demonstrates that the FCS autocorrelation functions for the PS-264/17 and PS-17/17 samples coincide provided the time is multiplied by the factor $\alpha=7.2$ in the case of the PS-17/17 sample. This scaling is valid for all concentrations under consideration because the $17 \mathrm{~kg} / \mathrm{mol}$ matrix PS chains do not form an entangled network in semidilute solution as mentioned earlier. Hence the polymeric nature of these short matrix chains does not lead to additional characteristic features of the FCS autocorrelation functions. In the case of a similar comparison of the FCS autocorrelation functions for the PS-264/67 and PS-67/67 samples, scaling can be found only for concentrations lower than $c^{+}$. For higher concentrations deviations from a simple scaling law are found because the self-diffusion coefficient and the cooperative diffusion coefficient exhibit different dependencies on the molecular weight [2].

\section{Conclusions}

Fluorescence correlation spectroscopy has been used to study the dynamics of labeled tracer polystyrene chains in a system consisting of matrix polystyrene chains dissolved in toluene [figure 1]. The self-diffusion coefficient of the tracer polystyrene chains has been measured for arbitrary concentrations of the matrix polystyrene chains. Moreover, the cooperative diffusion coefficient has been determined in the semidilute 

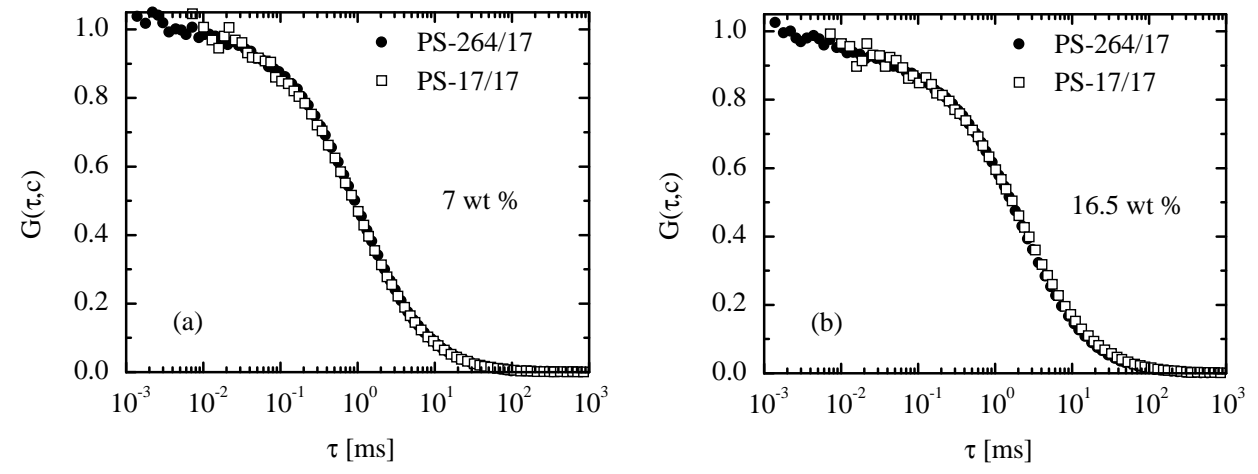

Figure 4. Normalized FCS autocorrelation functions for the samples PS-264/17 and PS-17/17 (see table 1). The concentration of the $17 \mathrm{~kg} / \mathrm{mol}$ matrix PS chains is 7 wt \% in panel (a) and $16.5 \mathrm{wt} \%$ in panel (b). The autocorrelation functions of both samples coincide for a given concentration because the time $\tau$ for the PS-17/17 sample has been multiplied by the factor $\alpha=7.2$ as is discussed in the main text.

entangled concentration regime due to the transient entanglement network [figure 2 . The minimum concentration of matrix polystyrene chains at which the cooperative diffusion coefficient can be detected by FCS is independent of the molecular weight of the tracer polystyrene chains [table 1. It has been suggested earlier in the context of polymer fiber spinning that a polymer solution is converted to a more stable elastically deformable network at such a concentration [30, 31, 32, 33, 34. Due to the resulting effective longe-range interaction of the polymer chains, the cooperative diffusion coefficient can be detected by FCS even in the case that the number of labeled polymer chains is considerably smaller than the number of unlabeled polymer chains. A theoretical description of the diffusion coefficients is given by a Langevin and integral equation theory [figure 3. Moreover, a single master autocorrelation curve has been found for short unentangled polystyrene matrix chains [figure 4 .

In summary, the present work gives further support to the recent conclusion that both the self-diffusion coefficient and the cooperative diffusion coefficient can be obtained experimentally using the same technique [2].

\section{References}

[1] Doi M and Edwards S F 1986 The Theory of Polymer Dynamics (Oxford: Clarendon Press)

[2] Zettl U, Hoffmann S T, Koberling F, Krausch G, Enderlein J, Harnau L and Ballauff M 2009 Macromolecules 429537

[3] Zettl H, Häfner W, Böker A, Schmalz H, Lanzendörfer M, Müller A H E and Krausch G 2004 Macromolecules $\mathbf{3 7} 1917$

[4] Zettl H, Portnoy Y, Gottlieb M and Krausch G 2005 J. Phys. Chem. B 10913397

[5] Zettl H, Zettl U, Krausch G, Enderlein J and Ballauff M 2007 Phys. Rev. E 75061804

[6] Magde D, Elson E and Webb W W 1972 Phys. Rev. Lett. 29705

[7] Rigler R and Elson E S 2001 Fluorescence Correlation Spectroscopy: Theory and Applications (Heidelberg: Springer)

[8] Liu R, Gao X, Adams J and Oppermann W 2005 Macromolecules 388845

[9] Harnau L, Winkler R G and Reineker P 1996 J. Chem. Phys. 1046355

[10] Harnau L, Winkler R G and Reineker P 1998 J. Chem. Phys. 1095160

[11] Harnau L, Winkler R G and Reineker P 1999 Phys. Rev. Lett. 822408

[12] Winkler R G, Keller S, Rädler J O 2006 Phys. Rev. E 73041919 
[13] Shusterman R, Gavrinyov T and Krichevsky O 2008 Phys. Rev. Lett. 100098102

[14] Wachsmuth M, Waldeck W and Langowski J 2000 J. Mol. Biol. 298677

[15] Weiss M, Elsner M, Kartberg F and Nilsson T 2004 Biophys. J. 873518

[16] Masuda A, Ushida K, Okamoto T 2005 Phys. Rev. E 72 060101(R)

[17] Banks D S and Fradin C 2005 Biophys. J. 892960

[18] Reitan N K, Juthajan A, Lindmo T and de Lange Davies C 2008 J. Biomed. Opt. 13054040

[19] Cherdhirankorn T, Floudas G, Butt H-J and Koynov K 2009 Macromolecules 429183

[20] von Meerwall E D, Amis E J and Ferry J D 1985 Macromolecules 18260

[21] Schweizer K S and Curro J G 1997 Adv. Chem. Phys. 981

[22] Harnau L 2001 J. Chem. Phys. 1151943

[23] Schulz G V and Hoffmann M 1957 Macromol. Chem. 23220

[24] Fuchs M and Schweizer K S 1997 J. Chem. Phys. 106347

[25] Daoud M, Cotton J P, Farnoux B, Jannink G, Sarma G, Benoit H, Duplessix R, Picot C and de Gennes P G 1975 Macromolecules 8804

[26] Harnau L 2008 Molec. Phys. 1061975

[27] Bolisetty S, Airaud C, Xu Y, Müller A H E, Harnau L, Rosenfeldt S, Lindner P and Ballauff M 2007 Phys. Rev. E 75 040803(R)

[28] Bolisetty S, Rosenfeldt S, Rochette C N, Harnau L, Lindner P, Xu Y, Müller A H E and Ballauff M 2009 Colloid. Polym. Sci. 287129

[29] Kanematsu T, Sato T, Imai Y, Ute K and Kitayama T 2005 Polymer J. 37, 65

[30] Schreiber H P, Rudin A and Bagley E B 1965 J. Appl. Polym. Sci. 9, 887

[31] Hayahara T and Takao S 1967 J. Appl. Polym. Sci. 11, 735

[32] McKee M G, Wilkes G L, Colby R H and Long T E 2004 Macromolecules 37, 1760

[33] Shenoy S L, Bates W D, Frisch H L and Wnek G E 2005 Polymer 463372

[34] Weitz R T, Harnau L, Rauschenbach S, Burghard M and Kern K 2008 Nano Lett. 81187 